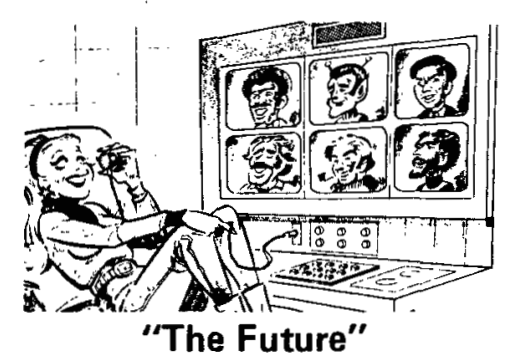

\title{
Information and Communication in the Third Millenium'
}

\author{
Edward C. Posner
}

\section{The variety of communication services, as well as the quantity of traffic, will have greatly increased by 2001 .}

\begin{abstract}
This paper presents the author's views on what the information and communication industry will be like at the start of the third millennium, with emphasis on the implications to information and communication theorists. The main conclusions are that communication will play a greater role in Society in the third millennium. Communication Theory will be more concerned with channel complexity than with channel capacity. More communication theorists will be needed, and they will be more broadly trained.
\end{abstract}

\section{INTRODUCTION}

This paper consists of an amplification of the author's talk of the same title given at the Communications Society Communication Theory Workshop held in April 1978. Although the views presented are the somewhat idiosyncratic ones of the author, they have nevertheless benefited from critical review and comments by the author's students at Caltech and numerous colleagues at JPL. Feedback from the Workshop participants has also influenced and improved this paper. Names of those who most moderated the views are omitted. I accept responsibility for the admittedly rose-colored picture given here.

\footnotetext{
IIf there is one.
}

The author is with the Department of Electrical Engineering and Jet Propulsion Laboratory, California Institute of Technology, Pasadena, CA 91125.
We will start with a look at information and communication at the start of the third millennium. ${ }^{2}$ This world view has some implications for workers in information and communication theory, as well as for everyone else. However, everyone else can dream as they wish, for we will only consider implications for the former group. We then look at some potential breakthroughs in information and communication theory that will either help all this happen or at least track the progress as it happens. Finally, we shall reach some conclusions; but these will be narrowed to the interests of information and communication theorists. It is recognized, however, that the most important implications of information and communication in the third millennium are not those for the IEEE Communications Society, but rather those for the Society at Large. ${ }^{3}$

\section{THE INFORMATION AND COMMUNICATION ENVIRONMENT AT THE START OF THE THIRD MILLENNIUM}

Let us take a look at information and communication at the start of the third millennium from the standpoint of the communication technologist. The first phenomenon may run against conventional wisdom. It is that guided waves, especially fiber optics, will replace satellites for fixed services such as point-to-point trunking.

\footnotetext{
2i.e., in 2001.

${ }^{3}$ Membership applications are available from the author.
} 
The cost and quality of fiber optics and the technology to distribute it plus the demand for orbital space are two salient trends. These trends will have meant that by the start of the third millennium, guided waves will carry the wideband aggregated common carrier communications now handled by satellite links. This even applies across oceans, not only across continents.

Guided waves will carry the wideband
aggregated common carrier communications
now handled by satellite links.

Is this bad for the communications and observation satellite industry? No-on the contrary, the equatorial belt at synchronous altitudes will be saturated with all kinds of satellites, including ones for mobile communications. However, a higher density will be tolerable due to such techniques as polarization isolation, beam shaping, and spread spectrum.

Mobile communications will be greatly expanded (see Fig. 1). This includes the use of satellites for communication with ships, aircraft, and other moving platforms. All automobiles will be equipped with true communication devices for voice and traffic control. Rediffusion points within the city will maintain continuous contact, with most signals distributed by guided waves up to the rediffusion points. However, we shall shortly see that commuter automobile traffic will be starting to decrease, because communications into homes and offices will decrease the need for it. The military will be a heavy user of mobile services, including man-pack field telephones. However, human-portable high-quality voice communications will not yet have invaded the civil sector, if it ever will be widespread for that use.

Citizens band will remain at the recreational level, and not evolve into a true communication system. Its function as a communications service will be completely taken over by mobile telephony. The manufacturers are likely to be very different, because mobile telephony is a system and citizens band is not.

All this mobile and military communication will greatly increase the radio frequency interference (RFI) seen by fixed and mobile services. Fig. 2 shows a telemetry spectrum of today being disturbed by two RF interferers. In the future, we may see dozens of interferers in the same spectrum, although spread spectrum may reduce the peaks and fill in the valleys. While transmitter, receiver, and antenna technology will offer some slight relief, the main technological result of such interference will be the development of increasingly sophisticated monitoring instruments. They will be based on high-accuracy highresolution wideband spectrum analyzers. Individual users will have their own spectrum analyzers if their volume of communications justifies it, but more importantly, the Federal Communications Commission (FCC) will have them. Automatically operated RFI monitoring stations will be deployed by the thousands all over the United States and much of the world. As we shall see, the role of the FCC will be much more in the monitoring aspect than in the regulatory aspect.

All this RFI will make deep space communication inoperable at microwave frequencies, whether on Earth or in Earth orbit. This is because of the extreme sensitivity required for communication from the edge of the Solar System and beyond. Millimeter waves will offer relief in orbit, because of the shielding provided by the Earth's atmosphere and the higher antenna directivities obtainable. Fig. 3 shows a concept of such an orbiting deep space relay station. Relay to Earth will still be at microwave frequencies, but the stronger signals available for such relay are more compatible with the forecast RFI environment.

When we said "at the edge of the Solar System and beyond," we meant it. The first spacecraft, powered by a nuclear rocket, will just be leaving the Solar System to explore the interstellar environment at low data rates-

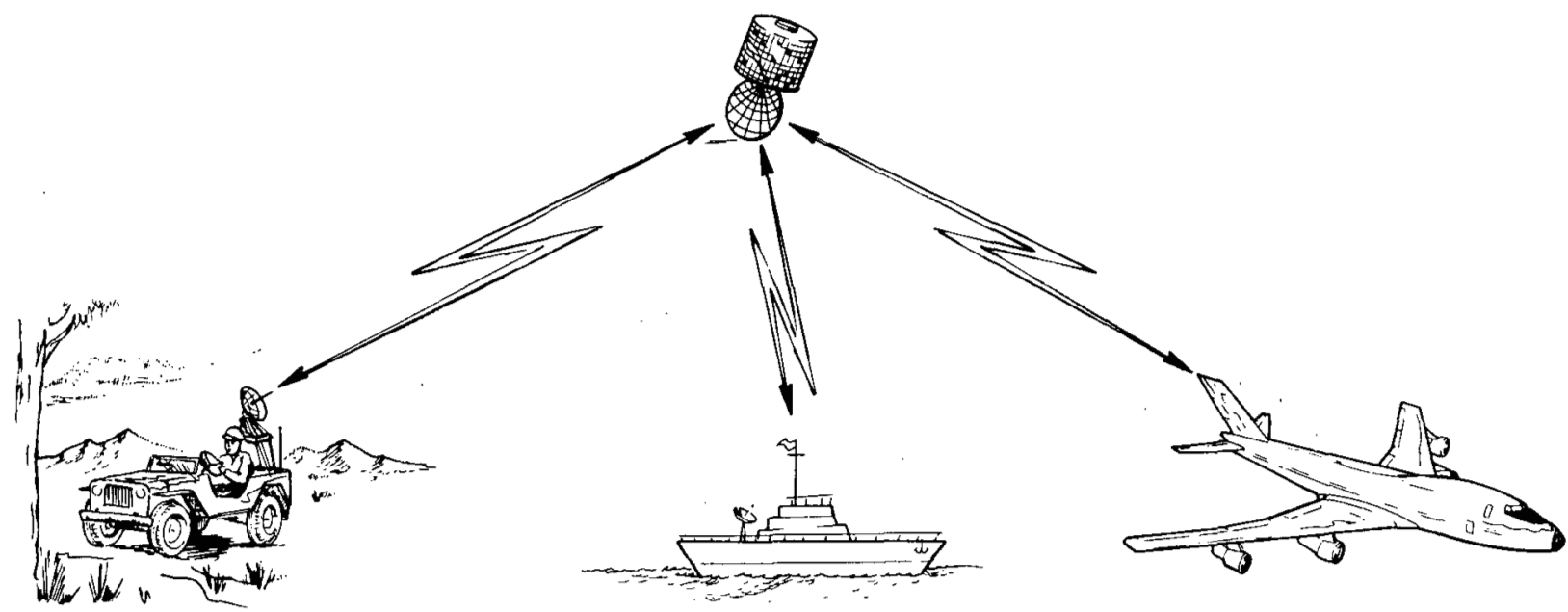

Fig. 1. Mobile communications. 


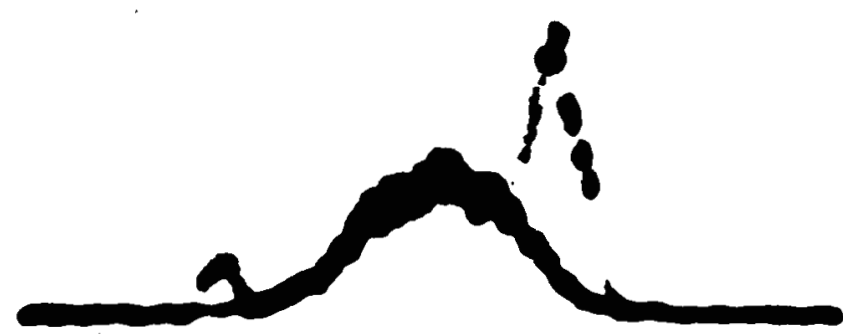

Fig. 2. Spectrum with RFI.

ultimately very low. The Doppler shifts will be so large that frequency bands will have to be changed during the flight several times. This will be accomplished by changing the feed and low noise amplifiers in the orbiting relay satellite. The first such frequency change will not yet have been made by 2001 .

Meanwhile, back on the inner planets, a Manned Mars Mission will finally be operational. There will be highrate communication both ways. The uplink will be used, among other things, to send Earth-generated video to the astronauts on Mars. Super Bowl XXXV4 will not be missed by the astronauts just because they are on Mars.

${ }^{4}$ Final score Mexico City 17, Tokyo 14, in overtime. It will be played in the Coliseum (in Rome).
On another inner planet, communication through the ocean to submerged moving platforms such as submarines, mining explorers, and teleoperators will have become routine at moderate rates. The blue-green window of seawater will be the medium for this laser-based capability.

On the landlocked side, information networks will infiltrate all offices and most homes. This includes two-way video, about which more will be said shortly. Many advances in the use of computing for such things as electronic funds transfer (EFT) will depend rather more on communications than on computing.

For example, paying one's monthly bills will not be automatically done by prior authorization, with one bank's computers getting paid from another bank's computers with no intervention by the person paying the bills. People won't go along with this-they want to be involved in the transfer of money from their account to the creditor's account. We will have EFT, but with the person directly involved in the link (see Fig. 4). Readers of this article and their children will review the month's bills on the terminal and themselves authorize payment from the terminal. A message management network will transfer the correct messages to each bank (payer's and payee's) involved, and initiate the computing which affects the

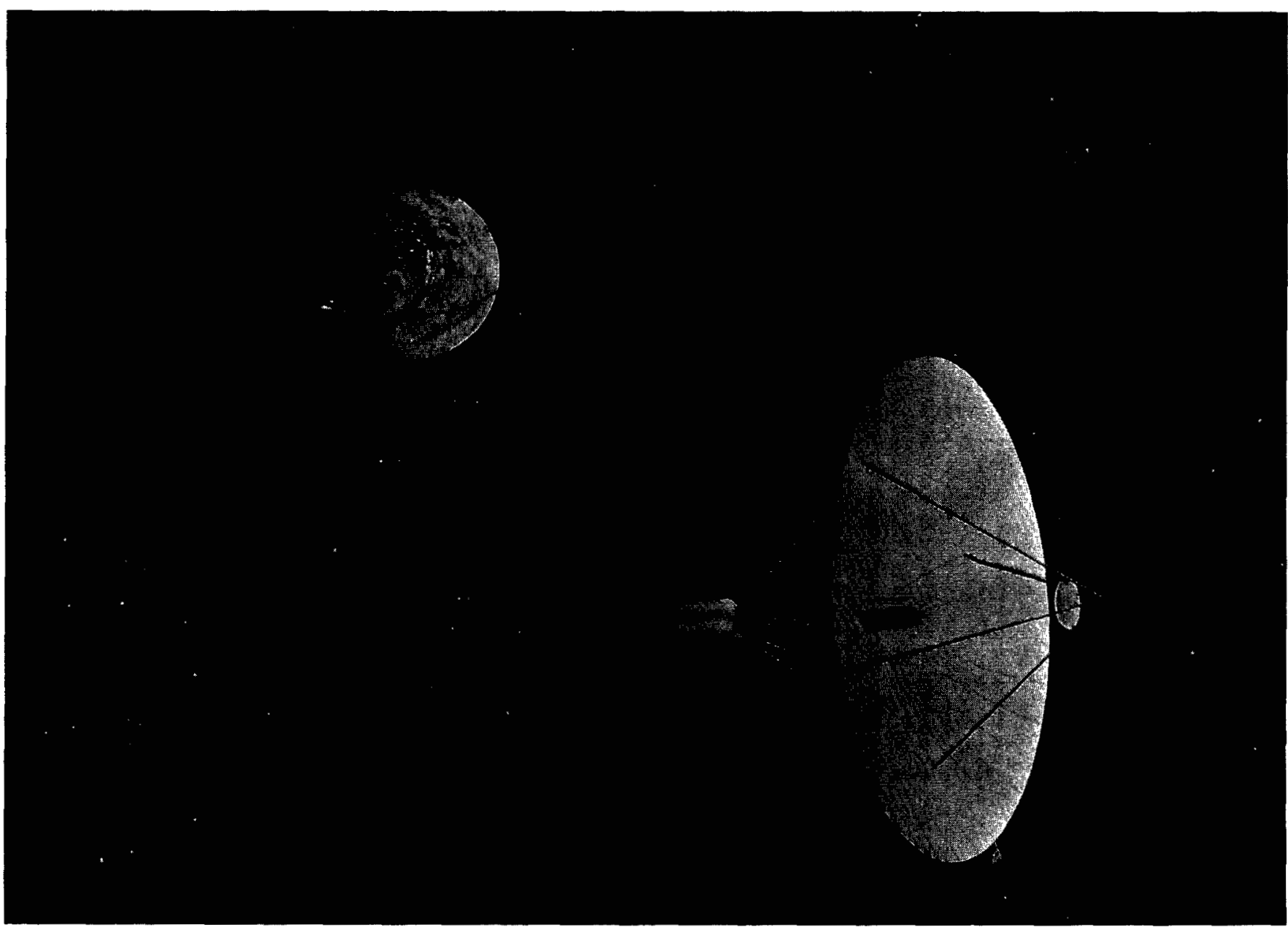

Fig. 3. Orbiting Deep Space Relay Station. 


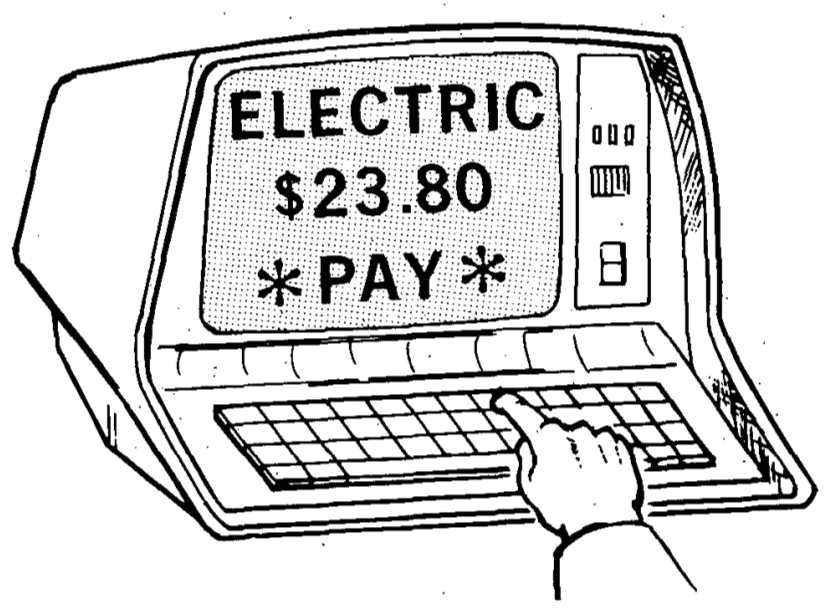

Fig. 4. Electronic funds transfer (EFT).

funds transfer. The individual's own record of transactions will be stored in his local data base for comparison with bank statements transmitted at periodic intervals.

If you can pay your bills without leaving the house, even to mail checks, and without the need for paper, what else can you not do? Indeed, many types of employment will be decentralized. The information networks we have been talking about will permit this. Networks and terminals will be so easy for people to use that most clerical and intellectual occupations will be dispersed-if not into homes then at least into a multiplicity of rather small department and subdepartment offices, as shown in Fig. 5. This further increases the reliance of society on communications, but opens up possibilities of more varied life styles without requiring people to drop out of the mainstream. It also makes it easier for small companies to compete with the biggies by allowing national marketing from remote business centers.

Along these lines, technical meetings will, in many instances, take place over an information network. In fact, the Communication Theory Workshop in 2001 will actually be held in a very large-scale integrated. (VLSI) chip in a Chicago switching center. The IEEE telepoker network will be used, combined with EFT, to conduct the annual game. The rate is measured in bets/second-a bet is two bits. ${ }^{5}$ In each instance of dispersed meetings, a trade-off has to be made between communication cost and quality as against transportation costs. However, fiber optic video links are expected in many instances to tip the balance in favor of communication over transportation.

By 2001, the VLSI revolution will at last be nearing its end. We will see some more of the results below-intelligent networks with information as well as data processing. In fact, much of the interactive multiterminal communication traffic we have been talking about will be routed through networks with delay and computation at the nodes. As one example of what we mean by infor-

${ }^{5}$ Shannon's limit is higher than this. mation as opposed to data processing, one will be able to route a call or electronic message to a known person without necessarily having to know the equivalent of his "telephone number". One will be able, for a price, to conduct a dialogue over the terminal with an intelligent network that will in effect give one the number or address. No more information operators (and no more recordings of the message that "someone will be here to help you!"). And information library services of a more general nature will be available with the same technology.

Commercial video broadcasting over the air will be almost entirely replaced by scheduled, or even on demand, transmission into homes via fiber optics. The price will be higher for demand transmission of say the equivalent of a Broadway play. The demand is initiated from the terminal and, as are all bills, the bill is paid monthly by the interactive EFT we have discussed. A very wide vari- . ety of programming will exist, and the advertising monopoly of network television will be broken. This will also make it easier for new firms or local products to compete. Diversity of opinion and the equal access of ideas will increase. As an example, in metropolitan areas, political discussions can take place on issues concerning areas smaller than the entire metropolitan area. Local studios (Fig. 6) will be plentiful. As one instance, two-way distribution to and from the residents within a single city

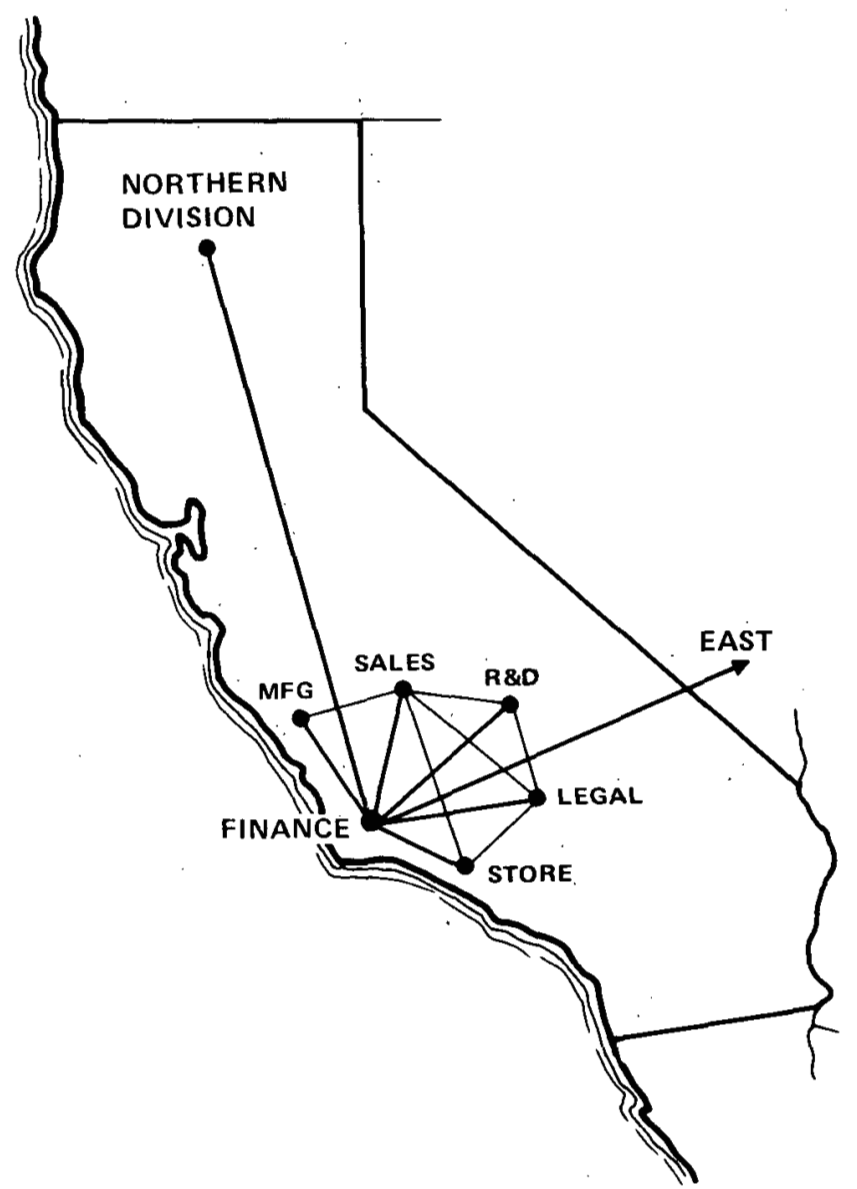

Fig. 5. Dispersion of company departments. 


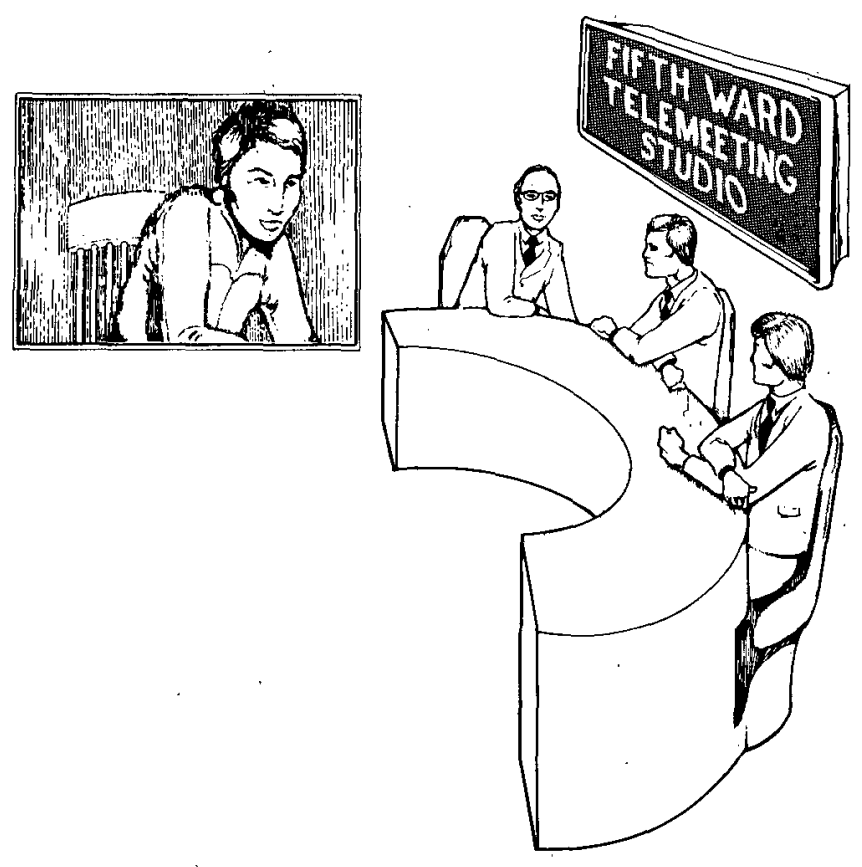

Fig. 6. Local telemeeting studio.

ward wil be easy to arrange. Metropolitan areas will still exist, because the trend to decentralization will not yet have completely changed residential patterns.

Because of the lessened reliance on over-the-air transmission and because of general pressure for more freedom, Government regulation of the communications and computing industry will decrease, at least in the United States. In fact, the trend to guided waves will once again restore constitutional protection to electronic messages. The residual Government regulation will involve frequency assignments for the mobile services we have discussed, and regulations attempting to protect the privacy and integrity of personal data in data bases.

\section{The electronic in-basket and electronic newspapers will mean the end of the postal service as we wish we knew it:}

We have alluded above to voice communications over networks, i.e., telephones, but actually many uses of the telephone will be replaced by an electronic in-basket that provides message forwarding, editing, retrieval, and redirection services. The Bell System will still exist, but will only be one carrier of several, depending on the kind of service. In any case, most terminals will be procured separately from the carriers, which will be mainly transmission companies or information services, depending on what they feel like calling themselves.

Newspapers and magazines, particularly specialized ones, will still exist. However, by 2001, a trend will have been set which will lead to their ultimate redefinition as electronic services instead of as pieces of paper. Inex- pensive graphics terminals in the home will permit this new kind of publication. In some cases, hard copy will be available, although with local disk storage this will not be so necessary. The electronic in-basket and electronic newspapers will mean the end of the postal service as we wish we knew it. It, or more efficient competitors, will be restricted to packages. The problem of electronic junk mail (EJM) can be solved by each individual programming his or her own terminal to reject unwanted commu. nications as they see fit.

With all these messages rattling around the world, security of communications in the civil sector will become absolutely essential. However, such security will be routinely available by the use of public-key cryptosystems and related technology. The eavesdropping industry is not, of course, going to go out of business. Instead, it will be focused on breaking down doors, not codes. The codes will be unbreakable. But it has always been true that one look is worth two wiretaps anyway.

We have painted a rather rosy picture of information and communication at the start of the third millennium, at least rosy for those in the industry and for believers in a Jeffersonian democracy. Now let's look at the implications for practitioners of the theory of information and communication.

\section{IMPLICATIONS FOR INFORMATION AND COMMUNICATION THEORISTS ${ }^{6}$}

We have seen in the preceding section that there will be an ambitious program of deep space exploration going on, including missions to the stars. So decibels on the Gaussian channel, the favorite of communication theorists, will still be important, at least for deep space. As we shall see, for communication in general, other types of problems will become dominant.

The trend to guided wave communication will mean that in many instances, bandwidth occupancy will no longer be the problem it has been. However, because of the wide use of mobile communications, there will still be a need for bandwidth-conserving modulation, data compression, and like techniques. This will be most true for mobile voice communication. Bandwidth conservation will, however, be less important in 2001 than at present. Wideband guided wave channels will be abundant, and the classical communication theorist's decibel and bandwidth will be less of a problem for such channels.

This is not so, as we have said, for mobile channels. For these, RFI may be even more constraining than decibels or bandwidth. Channel models incorporating RFI will therefore be necessary. Channel models for mobile communications in general will take on greater importance, particularly for undersea and urban channels. These models will, as we all know, differ greatly from the model of the classical Gaussian channel.

We have seen that cryptographic protection will be necessary if the information and communication revolu-

If there are any. 
tion is going to succeed. The theory of cryptography needs to be incorporated into the mainstream of communication theory so that it can be taken into account in the design process at an early stage.

More generally, even, the sources of data, and the subsequent processing of data to information by our intelligent networks, need to be modeled. Not only do we need to take cryptographic protection into account, we also need to accommodate the complexity of computing and the use of information if we, as theorists, are going to in fluence the evolution of our technology. This applies to everything from spacecraft to the Sears catalogue. The general message is that end-to-end information (rather than data) systems will be designed, and the theory should accommodate and facilitate that design process.

\section{End-to-end information (rather than data) systems will be designed.}

Information and communication theorists have not considered communications traffic to be part of communication theory, at least until very recently when packet switching networks began capturing the fancy of some of us. But models of communication traffic will be very important for the end-to-end design of information systems. In particular, we need models and theories of traffic for multiple data types, i.e., voice, video, and various kinds of digital computer and telemetry data. We also need to model the effect of computation and delay at various parts of the network. We should incorporate the generation of messages transmitted by nodes because of messages received by nodes. For example, this concept includes a message which requests transmission of items from a remote data archive. Traffic will mean more than Erlangs, just as communication will mean more than $\mathrm{dB}$ and $\mathrm{Hz}$.

The picture painted above indicates that the constraints on communication performance will be more in the human-network interface, in computing, and in signal and message processing, switching, and storage rather than in the classical communication parameters we have become used to since the late 40's. If these are the constraints, we need to have a theory to go along with the constraints.

The general conclusion, then, is that the variety of communication services will have greatly increased by 2001 , as well as the quantity of traffic. The overriding implication for information and communication theorists is that we will have to enlarge our domain of interests if we are to provide the service that the information and communication industry expects us to perform. That service is simple: to provide the firm basis for the principles of engineering design that are used to generate the information and communication systems of the year 2001.

Now that we have seen what the scenario of information and communication at the start of the third mil. lennium implies for information and communication theorists, let us look at some potential breakthroughs that would allow us to fulfill our function as the generators of the principles of end-to-end information system design.

\section{POTENTIAL BREAKTHROUGHS IN INFORMATION AND COMMUNICATION THEORY}

A first breakthrough in information and communication theory would merge the evolving theory of computational complexity with Shannon theory. The goal would be to rigorously define the fundamental limits to communication performance, taking into account source, channel,--and this is the new part-processing complexity. We can see some foreshadowings of this already. As an initial test of the new concept, we should obtain a precise result for the binary symmetric source and channel. This result would give the lowest output bit error probability attainable as a function of the rigorously defined implementation complexity of the encoder and decoder. More general sources and channels would be also incorporated into the theory, but quantitative answers would be harder to obtain at first.

A second breakthrough would involve a unified theory of multi-user communication networks of the kind illustrated in Fig. 7. Again, this is already foreshadowed in existing work. In particular, the theory would give permissible communication rates, error probabilities, and message delays as a function of sources, sinks, channels, interference (such as RFI), delay at nodes, storage, switching, and link reliability. We will want to incorporate processing complexity, but this addition will have to wait until complexity theory and Shannon theory have been merged for a decade or so.

A third breakthrough is to formally incorporate cryptographic protection into information and communication theory. Rigorous bounds on the degree of protection available are required, with emphasis on the limits to protection implied by constraints on complexity. The goal is to provide proveable protection at a desired communication rate, error probability, and encoder and decoder complexity. This theory can later be integrated into the theory of multi-user communication networks described above.

A fourth breakthrough involves a theory of interactive information traffic in networks. There is a need for a formal theory of what traffic levels are supportable with what networks and with what diversity of sources. Such a theory would make long-term capability planning easier. Delay and complexity should be incorporated. Moreover, the possibility of information generation, processing, and forwarding based on received messages should be specifically formalized. The theory ought to be strong enough to permit optimum network topologies to be derived, including assignment of complexity resources to nodes and capacity resources to links.

A fifth breakthrough is the modeling of humans as communication and information elements, as shown in 


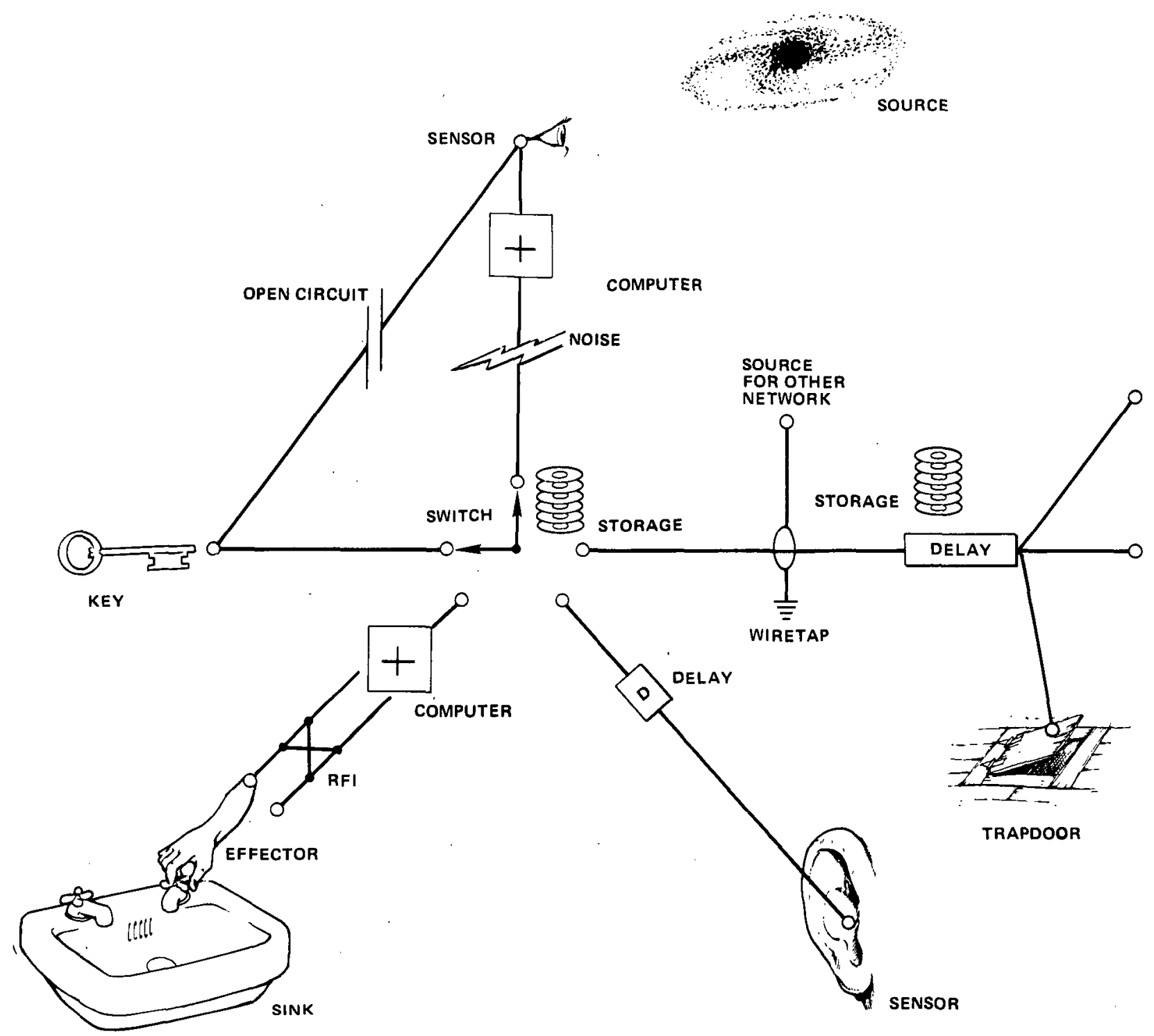

Fig. 7. Mulłl-user communication network.

Fig. 8. In fact, the distinction between data communication and processing and information communication and processing is that, in the latter, the human is part of the system. Such a mathematical model that could be used in design and evaluation might extend various existing psychophysical models such as the theory of memory chunks. If we model everything else, we should model the human as well, for taking our own capabilities into account could lead to information systems which are far more comfortable to use and adapted to our needs. This breakthrough is perhaps the most presumptuous suggestion of this paper, but one which, if it occurs, will be most rewarding.

A sixth breakthrough, which in a way encompasses all the others, involves a theory of the end-to-end information system. Such a theory is necessary as a basis for the proper design of end-to-end information systems. In the 40's, detection theory did this for radar systems. Such a new theory would evolve from the present-day specification of data rate and error probability as engineering parameters. It would indeed incorporate most, if not all, of the above advances, plus influences from outside information and communication theory proper. The methodology supported by the theory will

\section{The distinction between data communication and processing and Information communication and processing is that, in the latter, the human is part of the system.}

make the process of information systems design more accessible to the practicing engineer. At the same time, this methodology will enrich the information and communication theory which gave it birth by suggesting still newer problem areas. An exciting prospect indeed for the year 2001 ! 


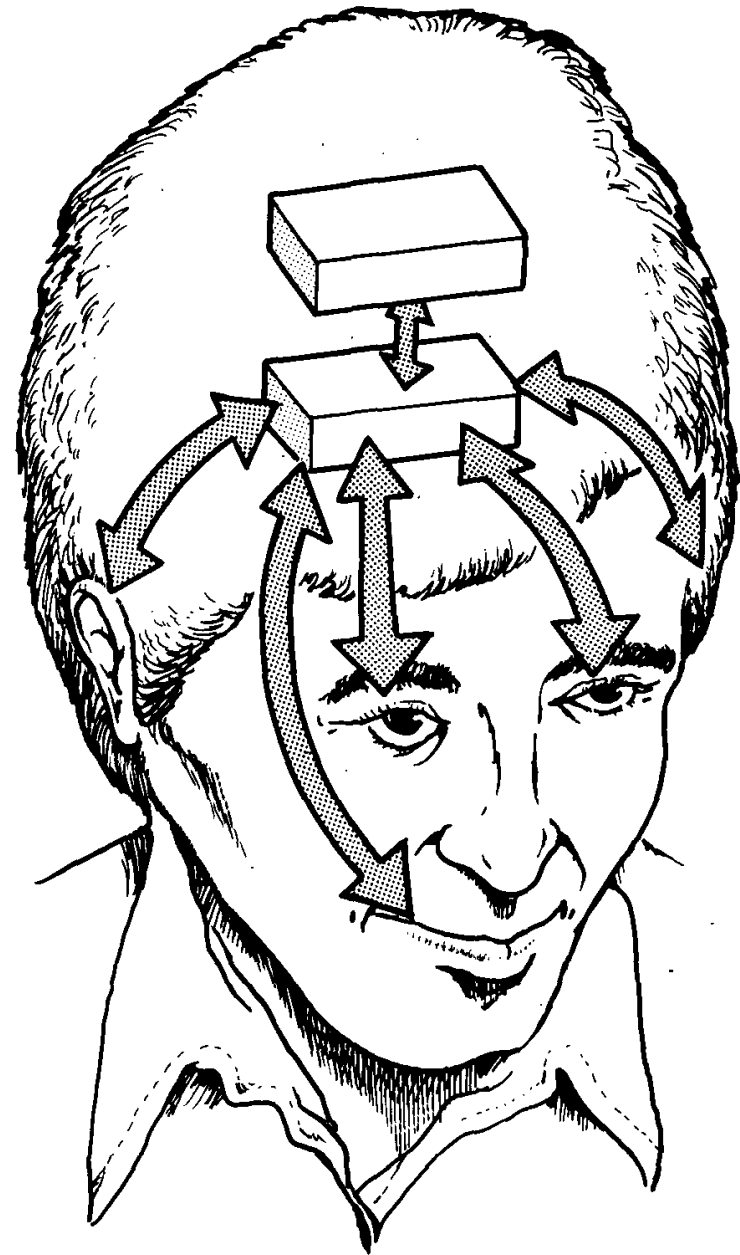

Fig. 8. Human as information processor.
We will need more information and communication theorists in the year 2001 than we need now; they will be more broadly educated and trained in an even wider variety of disciplines and technologies than they are trained in now.

evolution of information and communication technology we will have.

A fifth conclusion is that the human will become the subject of formal study and modeling by communication theorists. Complexity is a natural part of this study - the complexity of functions to be performed by people versus the functions to be performed by machines. This will mean still newer concepts for information and communication theorists to master.

The upshot of all this provides the sixth conclusion. We will need more information and communication theorists in the year 2001 than we need now. ${ }^{7}$ Not only that, but these theorists will be more broadly educated and trained in an even wider variety of disciplines and technologies than we are trained in now. This indeed may be the most exciting vista this paper has to suggest. And it offers the greatest challenge: how do we educate them--or ourselves? We will leave this for the Packets to the Editor Bin of the Communications Magazine.

${ }^{7}$ There is a hidden assumption here.

\section{CONCLUSIONS}

We can reach six conclusions based on the vision (or hallucination) suggested by what we have described above. First, it is clear that communication will play a greater role in society in the third millennium. Second, it. is apparent that a wider diversity of channel types will exist. Third, the major technology trends that influence the role and diversity of communication involve guided waves, especially fiber optics, plus interactive information networks incorporating very large-scale integrated circuits.

And we can reach a fourth conclusion: Communication theory will be more concerned with channel complexity than with classical channel capacity. The sooner we recognize this, the more influence over the

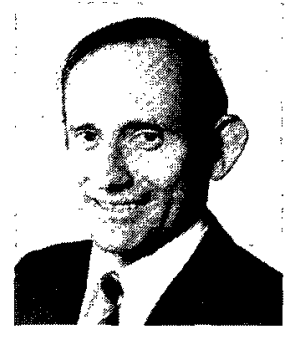

Edward C. Posner is Manager of the Tracking and Data Acquisition Planning Office at the Jet Propulsion Laboratory (JPL) and Visiting Professor of Electrical Engineering, both at the California Institute of Technology (Caltech). In, the JPL role, he is responsible for long-range planning for future deep space communication and tracking, with particular emphasis on the impact of new computer and communication technology. In particular, Dr. Posner has been leading a small group planing the future of information networks at JPL.

In his academic role, he has been organizing a Communications program within the Electrical Engineering Department, and has taught courses on information and communication theory and practice. He has co-authored a book with John R. Pierce based on one of these courses, entitled Introduction to Communication Science and Systems. It is due to be published by Plenum Press in 1979. Dr. Posner wishes to acknowledge the support of colleagues at JPL and the Campus in the preparation, review, and production of this paper, and welcomes comments from our readers. 